\section{L'Actualité économique}

L'ACTUALITÉ ÉCONOMIQUE

\section{Valeur de Shapley et partage équitable des ressources}

\section{Georges Zaccour}

Volume 64, numéro 1, mars 1988

URI : https://id.erudit.org/iderudit/601438ar

DOI : https://doi.org/10.7202/601438ar

Aller au sommaire du numéro

Éditeur(s)

HEC Montréal

ISSN

0001-771X (imprimé)

1710-3991 (numérique)

Découvrir la revue

Citer cet article

Zaccour, G. (1988). Valeur de Shapley et partage équitable des ressources. L'Actualité économique, 64(1), 96-121. https://doi.org/10.7202/601438ar
Ce document est protégé par la loi sur le droit d'auteur. L'utilisation des services d'Érudit (y compris la reproduction) est assujettie à sa politique d'utilisation que vous pouvez consulter en ligne.

https://apropos.erudit.org/fr/usagers/politique-dutilisation/ 


\title{
Valeur de Shapley et partage équitable des ressources
}

\author{
Georges ZACCOUR \\ École des Hautes Études Commerciales de Montréal*
}

\section{INTRODUCTION}

Depuis la parution en 1944 de l'oeuvre magistrale de von Neumann et Morgenstern, Theory of Games and Economic Behavior, la théorie mathématique des jeux n'a cessé d'entretenir des relations privilégiées avec certaines disciplines des sciences sociales, comme en témoigne la vaste littérature existante. De par la nature même de cette théorie, les applications les plus marquantes sont, le plus souvent, reliées à la modélisation des comportements, catégorisés en coopératifs ou non coopératifs, des agents économiques et politiques. Dans le cas coopératif, l'attention des théoriciens s'est surtout tournée vers la recherche de procédures d'arbitrage aux propriétés «souhaitables» qui visent à partager entre les joueurs les gains résultant de leur coopération.

Ce travail s'articule autour d'une de ces solutions et qui porte le nom de son concepteur la valeur de Shapley. Celle-ci a été adoptée par plusieurs auteurs pour allouer les ressources communes à un ensemble d'agents, problème fréquemment rencontré dans la réalité et faisant défi à plusieurs disciplines et champ d'études tels que l'économie, la comptabilité et la recherche opérationnelle, pour ne citer que les plus en vue.

Dans la deuxième section, on effectuera un tour d'horizon des notions essentielles de la théorie des jeux coopératifs, qui constitue notre cadre conceptuel pour approcher ce problème, en insistant sur la motivation et l'interprétation de la valeur de Shapley (VS).

La troisième section constitue une revue (non exhaustive) de la littérature des applications de la VS à ce problème de partage, classées selon les trois thèmes suivants :

(i) le partage des coûts d'investissements publics ;

* Groupe d'études et de recherche en analyse des décisions (GERAD).

J'ai bénéficié de discussions très utiles avec A. Hourie tout le long de ce travail. Je tiens à l'en remercier tout particulièrement. Je remercie également G. Côté, R. Loulou, P. Marcotte, C. Nappi et $\mathrm{H}$. Schleicher pour leurs commentaires. 
(ii) la tarification d'un service;

(iii) la comptabilisation des coûts conjoints.

\section{UN TOUR D'HORIZON DE LA THÉORIE DES JEUX COOPÉRATIFS ${ }^{1}$}

Comme son nom l'indique, un jeu coopératif met en situation un ensemble de joueurs pouvant conclure des ententes en vue de maximiser leur profit conjoint. On suppose donc qu'il n'existe pas d'obstacles juridiques, sociologiques, économiques ou psychologiques à la coopération. Celle-ci prend généralement la forme d'une coordination des stratégies ou d'un arrangement visant à partager les gains engendrés par le jeu. On suppose aussi que ces derniers sont évalués en termes monétaires, que tous les joueurs ont une utilité constante pour la monnaie, que les comparaisons interpersonnelles des utilités sont possibles et que les paiements latéraux sont permis ${ }^{2}$.

La théorie des jeux coopératifs, qui est un moyen formalisé pour analyser les situations d'interdépendance entre agents économiques, peut être aussi vue comme un ensemble de deux parties. La première se préoccupe de la description des règles du jeu et du processus de regroupement des joueurs, tandis que la deuxième est axée sur la recherche de solutions possédant certaines caractéristiques. Dans les paragraphes suivants, on procédera à une revue rapide des notions essentielles de ces deux pôles d'intérêt.

\section{A. La description d'un jeu coopératif}

Un jeu peut être décrit de trois façons différentes dépendamment du degré de détail souhaité dans la présentation des règles du jeu et des actions disponibles aux joueurs. Ainsi, la forme développée (ou extensive) procède à une description mathématique de ces règles et de la séquence dynamique complète des actions de chaque joueur. La forme normale se contente d'examiner les actions ou les stratégies des joueurs et des gains associés à chacune d'elle. Dans le contexte des jeux coopératifs, on est surtout intéressé par le processus de formation des coalitions entre les joueurs et, par conséquent, on a besoin d'évaluer la force potentielle de chacune d'elle. Cette façon de décrire un jeu a été fournie par un concept formulé par von Neumann en 1928 et appelé la fonction caractéristique $\mathrm{du}$ jeu.

Formellement, soit $\Gamma$ un jeu coopératif à $n$ personnes et $I=\{1, \ldots, n\}$ l'ensemble des joueurs. Un sous-ensemble $S \subseteq I$ est appelé une coalition. En permettant, par convenance, l'existence d'une coalition vide, il y en aura au total $2^{n}$ coalitions possibles. Soit $P(I)$ l'ensemble des parties de $I$, alors la fonction caractéristique est définie comme une fonction à valeurs réelles $v: P(I)$ $\rightarrow R$ telle que $v(S)$ représente le maximum de gain qu'une coalition peut garantir

1. Les livres de Ekeland [9], Jones [16], Luce et Raïffa [20] et Shubik [34] ainsi que les articles de Billera et al. [4] et de Haurie [14] m'ont été particulièrement utile pour rédiger ce chapitre.

2. Il existe une théorie des jeux coopératifs sans paiements latéraux (Aumann, 1969). 
à ses membres. von Neumann et Morgenstern ont interprété le terme garantir de la façon suivante : la pire situation qui s'offre à une coalition $S$ est que les autres joueurs $I \backslash S$ forment une anti-coalition dont l'objectif est de minimiser le gain de $S$. Dans ce cas, $v(S)$ est donné par:

$$
v(S)=\max _{x \in X_{S}} \min _{y \in X_{I \backslash S}} \sum_{i \in S} P_{i}(x, y)
$$

où $P_{i}(x, y)$ est le gain espéré du joueur $i$ quand la stratégie mixte $x \in X_{S}, y \in X_{I} \backslash S$ est employée.

Cette interprétation offre un avantage technique considérable dans la mesure où le problème de trouver $v(S)$ est réduit à celui de résoudre un jeu non coopératif entre $S$ et $I \backslash S$ et dont la procédure est bien connue. Néanmoins, et comme le soulignent McKinsey [21] et Luce et Raiffa [20], la fonction caractéristique ainsi définie fournit une représentation pessimiste de la réalité. En effet, elle postule toujours la formation de coalitions très antagonistes $(S$ et $I \backslash S)$ ce qui ne réflète pas nécessairement le processus de formation de coalitions observé chez des joueurs rationnels par définition.

La fonction caractéristique $v$ possède les deux propriétés suivantes :

$$
\begin{aligned}
& v(\phi)=0 \\
& v(S \cup T) \geq v(S)+v(T) \forall S, T \subseteq I, S \cap T=\phi
\end{aligned}
$$

Tandis que la première propriété ne fait que formaliser l'idée que le gain réalisé par une coalition vide est nul, la deuxième - la suradditivité - stipule qu'une coalition de joueurs est au moins aussi efficace que l'ensemble de ces sous-coalitions disjointes. Un jeu où $v$ est additive $(v(S \cup T)=v(S)+v(T))$ est dit inessentiel i.e. il n'y a aucun stimulant à la formation de coalitions de plus d'un joueur.

\section{A.1 Imputations}

Une imputation est un ensemble de $n$ valeurs numériques $\left(x_{1}, \ldots, x_{n}\right)$ représentant les gains des joueurs à l'issue du jeu et satisfaisant les deux conditions suivantes:

$$
\begin{array}{ll}
\text { (i) } & x_{i} \geq v\{(i)\} \\
\text { (ii) } & \forall i \in I \\
\sum_{i \in I} x_{i}=v(I) & \text { où } v(I) \text { est le gain total du jeu. }
\end{array}
$$

La première condition signifie qu'aucun joueur n'acceptera un partage lui attribuant moins de ce qu'il pourrait gagner en agissant seul (rationalité individuelle). Par ailleurs, un partage satisfaisant $\sum_{i \in I} x_{i}<v(I)$ ne sera pas accepté par les joueurs parce qu'il revient à gaspiller la quantité $v(I)-\sum_{i \in I} X_{i}$. D'autre part, si on suppose que $\sum_{i \in I} x_{i}>V(I)$ ceci reviendra, selon l'expression d'Ekeland [9, p.57], «à vendre la peau de l'ours avant de l'avoir tué » (la solution n'est pas réalisable). Donc, $\sum_{i \in I} x_{i}=v(I)$. Cette condition est appelée la rationalité collective. 
La rationalité individuelle étant un ingrédient de base de la théorie des jeux, et d'ailleurs de l'approche décisionnelle en sciences sociales en général, a permis à la première condition d'échapper à la critique qui s'est concentrée sur la deuxième. Luce et Raiffa [20] ont émis des réserves quant à la validité du passage de la rationnalité individuelle à la rationalité de groupe. Leur argument est que cette dernière n'est ni un postulat ni un résultat de la théorie des jeux coopératifs. D'autre part, si on admet cette hypothèse, pourquoi ne pas l'étendre à toutes les coalitions $S\left(\sum_{i \in S} X_{i} \geq v(S)\right)$ plutôt que de la réserver uniquement à la grande condition $I$ ? La raison est, comme nous le verrons plus loin, avant tout technique.

Pour récapituler, un jeu coopératif à $n$ personnes avec paiements latéraux est un triplet $\langle I, V, X\rangle$ où $I=\{1, \ldots, n\}$ est l'ensemble des joueurs, $V$ une fonction suradditive de $P(I)$ dans $R$ vérifiant $v(\phi)=0$ et $X$ est l'ensemble des imputations tels que $x_{i} \geq v(\{i\}), \forall i \in I$ et $\sum_{i \in I} x_{i}=v(I)$.

\section{B. Les solutions}

L'unicité d'une solution est toujours une propriété souhaitable et rassurante, surtout quand il s'agit de partager le gain d'un jeu entre les agents impliqués. En général, un jeu coopératif décrit par une fonction caractéristique n'engendre pas une imputation unique et, par conséquent, l'intérêt des théoriciens s'est tourné vers la recherche de moyens ou de procédures qui permettent, faute d'avoir une solution unique, d'exclure un certain nombre d'imputations sur la base de critères justifiés intuitivement.

Dans ce qui suit, j'exposerai uniquement les solutions qui ont, tout comme la valeur de Shapley, fait l'objet d'applications dans le domaine du partage équitable des ressources, ce qui me permettra de s'y référer à l'occasion.

\section{B.1 Le noyau}

Pour pouvoir écarter certaines imputations, il faudrait définir des critères qui font qu'une coalition va préférer une imputation à une autre. Intuitivement, il est clair qu'une coalition refusera une imputation particulière s'il existe une autre qui procurera à ses membres un gain supérieur. Formellement, on dit qu'une imputation yest dominé ${ }^{3}$ par $x$ si les deux conditions suivantes sont remplies :

$$
\sum_{i \in S} X_{i} \leq v(S) \quad \text { (faisabilité) }
$$

$$
x_{i}>y_{i} \quad \forall i \in S \text { (préférabilité) }
$$

L'ensemble des imputations qui ne sont dominées par aucune coalition est appelé le noyau du jeu. Une caractérisation des imputations (si elles existent) qui appartiennent au noyau est énoncée par le théorème suivant ${ }^{4}$ :

3. On dit aussi que l'imputation y est bloquée par la coalition $S$.

4. La démonstration se trouve dans Jones [16, p.201]. 
Théorème: Une imputation $x=\left(x_{1}, \ldots, x_{n}\right)$ appartient au noyau d'un jeu coopératif $\langle I, v, X\rangle$ si et seulement si :

$\sum_{i \in S} x_{i} \geq v(S) \quad \forall S \subseteq I$.

Le noyau, proposé par Gillies en 1959, a la (bonne) propriété de satisfaire toutes les coalitions dans la mesure où aucune d'elle ne peut augmenter son gain. Par contre, le noyau peut être vide ${ }^{5}$.

\section{B.2 La solution de von Neumann-Morgenstern}

von Neumann et Morgenstern ont proposé dans [37] un concept de solution qui s'articule autour de la notion de stabilité qui s'énonce comme suit:

Définition: Un ensemble d'imputations $V$ d'un jeu coopératif $\langle I, V, X\rangle$ est stable:

(i) si $x$ et $y \in V$ alors $y$ n'est pas dominée par $x$ et $x$ n'est pas dominée par $y$ (stabilité interne);

(ii) si $y \notin V$ alors il $\exists x \in V$ tel que y est dominée par $x$ (stabilité externe).

Une solution von Neumann-Morgenstern est tout ensemble stable. Généralement, cette solution n'est pas unique.

\section{B.3 Le nucléole}

L'idée du nucléole, solution introduite par Schmeidler [29] en 1969, «est de minimiser le mécontentement maximum ou, en d'autres termes, minimiser la plainte la plus criante» (Ekeland [9, p. 97]). Pour le définir rigoureusement, quelques préliminaires techniques sont nécessaires.

Soit $\prec$ et $\leqslant$ deux relations binaires sur $R^{P}(p \in N)$ définies par:

$$
\begin{aligned}
& x<y \leftrightarrow\left[E j \in\{1, \ldots, p\}: \quad\left(x_{i}=y_{i} \text { pour } i<j\right) \text { et } x_{j}<y_{j}\right] \\
& x \leqslant y \leftrightarrow[x<y \text { ou } x=y]
\end{aligned}
$$

La relation $\leqslant$ est une relation d'ordre total appelée ordre lexicographique.

D'autre part, on définit composante par composante une application $\theta: R^{P}$ $\rightarrow R^{P}$ dite de classement :

$$
\begin{aligned}
& \theta_{1}(x)=\max \left\{x_{j} / 1 \leq i \leq p\right\} \quad=x_{i_{1}} \\
& \theta_{1}(x)=\max \left\{x_{j} / 1 \leq i \leq p, \quad i \neq i_{1}, i \neq i_{2}, \ldots, i \neq i_{j-1}\right\}=x_{i_{j}}
\end{aligned}
$$

5. En effet, considérons le jeu à 3 joueurs dont la fonction caractéristique est :

$$
\begin{aligned}
& v(\{1,2,3\})=1 \quad v(\phi)=0 \\
& v(\{1,2\})=v(\{1,3\})=v(\{2,3\})=a \quad 0<a<1 \\
& v(\{i\})=0 \quad i=1,2,3
\end{aligned}
$$

Si $a>2 / 3$, le noyau est vide, car de quelque façon que l'on partage 1 , il y aura toujours deux joueurs qui auront moins que a à eux deux et qui formeront donc une coalition bloquante. (Exemple tiré de Ekeland $[9$, p.60]). 
Définition: On appelle nucléole d'un jeu coopératif $\langle I, v, X\rangle$ une imputation $\left(\overline{X_{i}}\right)_{i \in I}$ telle que, pour toute imputation $\left(x_{i}\right)_{i \in I}$ on ait :

$$
\theta(v-\bar{X}) \leqslant \theta(v-x)
$$

Si $V(S)-x(S)>0$, la coalition $S$ s'estimera lésée par l'imputation $x$, et bénéficiaire si $v(S)-x(S)<0 . v(S)-x(S)$ mesure le mécontentement de la coalition $S$ face à l'imputation $x$ et $\bar{X}$ minimise le mécontentement maximum.

Deux éléments rendent le nucléole particulièrement intéressant. D'une part, il est unique et d'autre part, si le noyau n'est pas vide, alors le nucléole appartient à ce dernier. Ceci permet de sélectionner en quelque sorte une imputation du noyau, s'il en contenait plusieurs. Dans la littérature économique portant sur le bien-être social, il existe une analogie au nucléole offerte par Rawls [25] qui propose, faute d'atteindre dans certains cas l'optimum de Pareto, de maximiser la fonction d'utilité de l'individu le plus démuni.

\section{La valeur de Shapley}

La valeur de Shapley diffère des autres solutions, exposées dans la section précédente, dans la mesure où elle consiste en une évaluation a priori du jeu plutôt que d'être le résultat du jeu. Cette caractéristique en fait une solution qui, en quelque sorte, dispense de jouer. Pour mieux saisir sa motivation, citons Shapley:

"In attempting to apply the theory [of games] to any field, one would normally expect to be permitted to include, in the class of «prospects», the prospect of having to play a game. The possibility of evaluating games is, therefore, of critical importance. So long as the theory is unable to assign values to the games typically found in application, only relatively simple situations - where games do not depend on other games - will be susceptible to analysis and solution.»

[31, p. 307]

Pour déterminer cette valeur, Shapley a adopté l'approche axiomatique qui revient à sélectionner, sur une base conceptuelle ou intuitive, l'ensemble de propriétés souhaitables qu'elle devrait avoir. Ce faisant, Shapley a énoncé trois axiomes et a démontré l'existence d'un vecteur unique $\phi(v)=\left(\phi_{1}(v), \ldots, \phi_{n}\right.$ $(v))$ qui les satisfait, où $\phi_{i}(v)$ est la valeur du jeu pour le joueur $i$. Les axiomes sont les suivants :

Axiome de symétrie: Pour tout automorphisme $\pi$ du jeu $\langle I, v, X\rangle, \phi_{i}$ $(v)=\phi_{\pi_{i}}(v) \quad \forall i \in I$ où $\pi$ est une permutation de $I$ dans $I$. Cet axiome signifie que la valeur attribuée au joueur $i$ ne dépend que de sa force stratégique (et non de son libellé).

Axiomed'efficacité : $\sum_{i \in I} \phi_{i}(v)=v(I)$

Cet axiome correspond à l'optimalité au sens de Pareto. 
Axiome de linéarité : $\mathrm{Si} v$ et $w$ sont deux fonctions caractéristiques en rapport avec le même ensemble $I$ de joueurs, alors

$$
\phi_{i}(v+w)=\phi_{i}(v)+\phi_{i}(w) \quad \forall i \in I
$$

La formule explicite de la valeur de Shapley est donnée par le théorème suivant :

Théorème ${ }^{6}$ : L'unique vecteur $\phi(v)=\left(\phi_{1}(v), \ldots, \phi_{n}(v)\right)$ qui satisfait ces trois axiomes est donné par:

$$
\phi_{i}(v)=\sum_{S \ni i} \frac{(n-s) !(s-1) !}{n !}(v(S)-v(S \backslash\{i\}))
$$

où $n$ et $s$ représentent respectivement le nombre total de joueurs et celui appartenant à la coalition $S$.

C'est une interprétation probabiliste qui a été donnée à cette valeur. En effet, supposons que $i \in S$. La coalition $S \backslash\{i\}$ aurait pu se former de $(s-1)$ ! façons et les autres joueurs $(n-s)$ peuvent être arrangés de $(n-s)$ ! façons. Donc, le nombre de façons que le joueur $i$ peut rejoindre la coalition $S \backslash\{i\}$ est de $(n-s) !(s-1)$ !. D'autre part, la grande coalition $I$ peut se former de $n$ ! façons. En supposant chacune d'elle équiprobable alors $\frac{(n-s) !(s-1) !}{n !}$ n'est rien d'autre que la probabilité que le joueur $i$ se joigne à la coalition $S \backslash\{i\}$. Par ailleurs, $(v(S)-v(S \backslash\{i\}))$ mesure la contribution du joueur $i$ à la coalition $S$ et, par conséquent, $\phi_{i}(v)$ représente une moyenne de ses contributions marginales à toutes les coalitions auxquelles il est susceptible d'adhérer.

L'unicité, la symétrie (signifiant que deux joueurs ayant la même force stratégique recevront le même gain) et l'efficacité rendent la valeur de Shapley particulièrement attrayante pour traiter le problème du partage équitable des ressources communes à plusieurs agents économiques. En effet, l'unicité est rassurante au niveau psychologique dans la mesure où l'agent n'aurait plus à se poser la question, à savoir s'il existe une meilleure répartition de ces ressources d'autant plus que celle-ci est Pareto-optimale (axiome d'efficacité) et équitable. Signalons que cette valeur existe même si le noyau est vide, et qu'elle coïncide avec son centre de gravité si le jeu est convexe ${ }^{7}$. D'autre part, si les deux premiers axiomes ont été accueillis favorablement, ce ne fut pas le cas de l'axiome de linéarité comme en témoigne la critique de Luce et Raiffa [20]. Leur argument est que si un jeu $u$ est composé de deux fonctions caractéristiques $v$ et $w(u=v+w)$, on ne peut pas s'attendre à ce qu'il soit joué comme s'il était la somme de deux jeux différents dans le sens où il aura sa propre structure qui déterminera les gains qui peuvent différer de $v$ et $w$.

6. Une démonstration détaillée de ce théorème se trouve dans Berge [2], Shapley [31] et Vorob'ev [38).

7. Un jeu est convexe si la fonction caractéristique satisfait la condition suivante : $v(S)+v(T) \leq v(S \cup T)+v(S \cap T) \quad \forall S, T \in P(I)$

Rappelons que Shapley [32] a démontré que le noyau d'un jeu connexe n'est pas vide. 


\section{C.1 Valeur des jeux non atomiques}

Aumann et Shapley [1] ont étendu cette notion de valeur aux jeux non atomiques (ou atomistiques), jeux caractérisés par la présence d'un continuum de joueurs (plutôt que d'un nombre fini) et dont les décisions prises par chaque joueur n'ont pas d'influence sur les autres. L'analogie avec le modèle microéconomique classique, où on postule la présence d'un nombre "élevé» de producteurs et de consommateurs, est claire. Dans le paragraphe suivant, je me contenterai d'introduire le minimum de préliminaires techniques nécessaires à la détermination explicite de la valeur des jeux non atomiques ${ }^{8}$.

Soit $(I, C)$ un espace mesurable, où $I$ est l'ensemble des joueurs et $C$ une $\sigma$-algèbre des sous-ensembles mesurables de $I$. Les éléments de $C$ sont appelés des coalitions. Un jeu $v$ sur $I$ est une fonction à valeurs réelles sur $C$ satisfaisant $v(\phi)=0 . v$ est un jeu monotone si $\forall S, T, \in C, S \subset T=>v(S) \leq v(T)$. Un jeu $v$ est à variation bornée s'il est la différence entre deux jeux monotones. L'espace de tous les jeux à variation bornée est appelé $B V$ (Bounded Variation).

Soit $Q$ un espace quelconque de jeux, $Q^{+}$désigne alors le cone des éléments monotones de $Q$. Une application est dite positive si elle renvoie $Q^{+}$dans $B V^{+}$. Le sous-espace de $B V$ formé des fonctions d'ensembles bornées et additives est appelé $F A$. Une mesure $\mu$ est dite non atomique si $\forall S \in C,|\mu(S)|>0=>$ E $T \subset S, T \in C$ et $0<|\mu(T)|<|\mu(S)|$. Soit $N A$ l'espace des mesures non atomiques sur $(I, C)$. Le sous-espace de $B V$ engendré par toutes les puissances de $N A^{+}$est appelé $p N A$.

D'autre part, soit $G$ le groupe des automorphismes sur $(I, C)$. Chaque $\theta \in G$ induit une application linéaire $\theta_{*}$ de $B V$ dans lui-même définie par $\left(\theta_{*} V\right)$ $(S)=v(\theta S), \forall S \in C$. Un sous-espace $Q$ de $B V$ est dit symétrique si $\theta * Q=Q$, $\forall \theta \in G$.

Pour terminer, soit $Q$ un sous-espace symétrique de $B V$. Une valeur sur $Q$ est alors une application linéaire positive $\phi$ de $Q$ dans $F A$ qui satisfait les deux axiomes suivants :

Axiome d'efficacité: $(\phi v)(I)=v(I) \quad \forall v \in Q$

Axiome de symétrie : $\phi \theta_{*}=\theta_{*} \phi \quad \forall \theta \in G$

La formule explicite de cette valeur est fournie par le théorème suivant :

Théorème: Il existe une valeur unique $\phi$ sur $p N A$. Soit $\mu$ un vecteur de mesures dans $N A$ et $f$ une fonction continuement différentiable sur le domaine $R$ de $\mu$ où $f(0)=0$. Alors, fo $\mu \in p N A$ et quand $R$ est de pleine dimension,

$$
\begin{aligned}
& \phi(f o \mu)(S)=\sum_{j=1}^{n} \mu_{j}(S) \cdot \int_{0}^{1} f_{j}(t \mu(I)) d t \\
& \text { où } f_{j}=\frac{\delta f}{\delta x_{j}}
\end{aligned}
$$

8. Les définitions et les notations sont exactement celles figurant dans Aumann et Shapley [1] et reprises intégralement par Billera et al. [4]. 
Cette formule met un point final à la revue des notions essentielles de la théorie des jeux coopératifs qui nous intéressent pour aborder le problème du partage équitable des ressources.

\section{LES APPLICATIONS DE LA VALEUR DE SHAPLEY}

Si la formulation de la valeur de Shapley (VS) remonte à 1953, ce n'est que durant les années soixante que les applications de cette solution (a priori) d'un jeu coopératif à diverses problématiques de l'univers des sciences sociales ont commencé à apparaître. C'est Shubik qui a montré la voie à suivre dans son article célèbre « Incentives, Decentralized Control, the Assignment of Joint Costs and International Pricing» paru en 1962 [33]. Dans cet article l'auteur propose la VS comme mécanisme de répartition du profit d'une organisation complexe entre ses départements et démontre, à travers une série d'exemples, comment cette façon de procéder constitue un stimulant à l'innovation. L'extension, par Aumann et Shapley [1], de la VS aux jeux non atomiques a permis d'aborder les problèmes où les agents sont infinitésimaux.

Dans cette section, on procédera à un parcours de la littérature portant sur les applications de la $V S$ aux problèmes de partage des ressources communes à plusieurs partenaires. Bien que toute cette littérature s'articule autour de la même préoccupation, la recherche d'une procédure de partage équitable, il serait plus clair de l'aborder selon les trois thèmes suivants :

(i) la répartition des coûts d'investissements publics ;

(ii) la tarification d'un service offert par un monopole public (ou privé dans certains cas);

(iii) la comptabilisation des coûts induits par des processus de production conjoints et la répartition des ressources à l'intérieur d'une organisation.

Dans les paragraphes suivants, on raffinera cette caractérisation de la littérature en exposant pour chacun des thèmes, (1) la nature et la motivation du problème considéré, (2) l'approche méthodologique qui a été à l'oeuvre pour le traiter et (3) les applications empiriques quand elles se présentent.

\section{A. La répartition des coûts d'investissements publics}

\section{A.1 Le problème}

Les économies d'échelle ou de coûts incitent les agents économiques à se regrouper pour réaliser certains projets. Ainsi, par exemple, la construction d'une centrale électrique pour satisfaire la demande de $n$ localités pourrait s'avérer plus profitable que de construire $n$ petites centrales chacune desservant une localité. Si tel est le cas, le problème qui se pose à l'économiste est alors de trouver une procédure lui permettant de répartir le coût global de la grande centrale entre les $n$ localités. Il est clair que tout partage devrait, pour qu'il soit 
acceptable, satisfaire un ensemble de propriétés découlant de considérations d'optimalité et de «bon sens». Pour les dériver, suivons le cheminement de Schotter et Schwödiauer [30].

Soit $N=\{1, \ldots, n\}$ l'ensemble des $n$ localisés ou de tout autre ensemble de décideurs (ou éventuellement de décisions). Un sous-ensemble $S \subseteq N$ est appelé une coalition. Soit $C(i)$ le coût encouru par la localité $i(i=1, \ldots, n)$ si elle construit sa propre centrale et $R(i)$ sa contribution si elle participe au projet conjoint (la grande centrale). On admet aisément que $i$ n'accepterait aucune procédure de partage des coûts qui ne satisfait pas:

$$
R(i) \leq C(i) \quad i=1, \ldots, n
$$

Cette condition qui a été appelée The stand-alone test par Faulhaber et Zajac [10] n'est rien d'autre qu'un énoncé du principe de la rationalité individuelle. Conceptuellement, il n'existe aucune objection à généraliser ce principe à toutes les coalitions et, par conséquent, il faudrait que la procédure de partage satisfasse la condition dite The generalized stand-alone test qui s'écrit:

$$
\sum_{i \in S} R(i) \leq C(S)
$$

$\forall S \subseteq I$

D'autre part, si on exige que les contributions des $n$ localités couvrent exactement le coût de l'investissement global $C(N)$, ce qui revient à supposer l'absence de subventions, alors:

$$
\sum_{i=1}^{n} R(i)=C(N)
$$

Selon la terminologie de la théorie des jeux coopératifs, tout vecteur satisfaisant les conditions (1) et (3) est appelé une imputation. Si cette dernière satisfait (2), elle appartient alors au noyau. La fonction caractéristique de ce jeu de partage des coûts est déterminée par la fonction $C(\cdot)$ qui, s'agissant d'un coût plutôt que d'un gain, est sous-additive. À partir de là, toutes les solutions d'un jeu coopératif, discutées auparavant dans un contexte théorique, sont applicables directement à ce problème. Sans éliminer totalement la possibilité de recourir au noyau ou au nucléole, je crois que la $V S$ présente un certain nombre d'avantages pour traiter ce type de problèmes. En effet, en plus d'être unique, contrairement au noyau qui contient généralement (s'il n'est pas vide) plusieurs imputations, la VS constitue une solution équitable, propriété d'une importance capitale dans un contexte de partage des coûts. Son avantage par rapport au nucléole, seule autre solution faisant référence à l'équité (Spinetto [36]) en minimisant la plainte de la coalition la plus démunie, est plutôt d'ordre opérationnel. En effet, il n'existe pas une formule mathématique, comme c'est le cas de la VS, pour calculer le nucléole qui s'obtient plutôt par la résolution d'une suite de programmes linéaires propres à chaque problème ${ }^{9}$.

9. Dans un article portant sur le problème d'allocation des coûts sur une structure d'arbre, Megiddo [22] a proposé un algorithme pour calculer la $V S$ et un autre pour le nucléole et a démontré que le premier converge en $\theta(n)$ opérations tandis que le deuxième prendrait $\theta\left(n^{3}\right)$ opérations. D'autre part, et toujours dans ce même contexte, la programmation du nucléole présente des difficultés algorithmiques disproportionnées par rapport à celles induites par la VS. 


\section{A.2 Quelques exemples d'applications empiriques}

Dans cette section, on passera en revue trois exemples d'application de la $V S$ à des problèmes réels de partage des gains résultant de la coopération de plusieurs joueurs.

\section{A.2.1 Un exemple de coopération régionale en matière énergétique}

Dans un article paru en 1974, Gately [11] propose de résoudre un problème de répartition des gains résultant d'une coopération entre trois régions en Inde pour planifier les investissements futurs en énergie électrique. À l'aide d'un programme mathématique, l'auteur estime les coûts d'expansion et de fonctionnement d'un système électrique pour chacune des cinq coalitions (non vides) possibles ainsi que les contributions individuelles sous les différentes hypothèses de coopération. La fonction caractéristique du jeu est obtenue en prenant la différence entre la somme des coûts individuels $C(i)$ sous l'hypothèse d'autosuffisance pour chaque région et la somme des contributions individuelles $R(i)$. Le gain de chaque joueur est donné par $P(i)=C(i)-R(i)$. Gately calcule ensuite le noyau pour déterminer l'ensemble des imputations acceptables. Vu que le noyau contient des imputations où le gain d'un (ou de plusieurs) joueur(s) peut être négatif pour des raisons découlant de l'optimisation d'une fonction objectif globale, l'auteur a développé, pour éliminer ces imputations qualifiées de non équitables, un ratio qui mesure la propension d'un joueur à empêcher la formation de la grande coalition qui s'avère la plus profitable (globalement). Pour le joueur $i$, ce ratio est égal à la perte encourue par les joueurs $j$ et $k$ quand $i$ refuse de collaborer sur la perte encourue par $i$. À partir de là, on peut éliminer les imputations pour lesquelles correspond au moins un ratio dépassant un seuil fixé arbitrairement (par exemple 2). Malgré ce tri, la solution du problème n'est toujours pas unique. C'est à ce moment que Gately se tourne vers, entre autres, la $V S$ qui, fait intéressant, appartient au noyau et d'autre part, les trois ratios (un par jour) de propension à perturber la grande coalition sont inférieurs à un i.e. chaque joueur perdrait plus que les deux autres réunis s'il décide de ne pas se joindre à eux.

\section{A.2.2 La coopération entre communes pour la construction d'une station d'épu- ration}

L'intérêt majeur du très long article (53 pages) de Schleicher [28] réside dans son analyse détaillée des techniques de répartition des coûts d'investissements publics inspirées des différentes solutions d'un jeu coopératif. Cette analyse est faite à travers le traitement d'un exemple mettant en jeu trois communes de tailles différentes qui déversent leurs effluents dans une rivière. Une pollution de celle-ci et de la nappe souterraine s'ensuit. Le problème est alors de déterminer équitablement la part de chaque commune dans la construction d'une station d'épuration. Plusieurs variantes de cet exemple sont étudiées et, notamment, les deux cas intéressants où (i) la $V S$ est dans le noyau et (ii) la $V S$ n'appartient pas au noyau. Dans le premier cas, la VS est considérée comme la solution du 
jeu d'autant plus qu'elle est équitable et acceptable (étant dans le noyau) par toutes les coalitions. Dans le deuxième cas, Schleicher décrète la supériorité de la VS par rapport aux imputations qui sont dans le noyau et propose, pour amener les joueurs qui pourraient être désavantagés, une panoplie de possibilités de taxation ou de subventions.

Un autre élément dans cet article qui mérite d'être relevé est l'interprétation différente donnée par l'auteur à la VS. En effet, dans la version fournie par Shapley lui-même, la VS évalue la moyenne des contributions marginales d'un joueur à toutes les coalitions auxquelles il est susceptible d'adhérer. Dans le cas d'un jeu à trois joueurs, il existe une interprétation équivalente qui consiste à évaluer «l'apport d'une coalition toute entière par rapport aux coûts indépendants de ses membres $S-\{i\}$ et de $\{i\}$, où $S-\{i\}$ est la sous-coalition la plus avantageuse. Ce gain net est réparti d'une manière égalitaire et affecté à $\forall i \in$ $S$ » $[28$, p. 1436]. Dans ce cas, la VS se calcule comme suit:

$$
\begin{aligned}
& C_{S}^{i}=[C(\{i\})-C(\{\phi\})] \text { pour } S=\{i\} \\
& i=1, \ldots, 3 \\
& \text { et } C_{S}^{i} \geq 0 \\
& C_{S}^{i}=\frac{1}{|S|}[C(S)-C(\{i\})-C(\{j\})] \text { pour } \underset{i \in S}{|S|}=2 \\
& j \in S \\
& i \neq j \\
& \text { et } C_{S}^{i} \geq 0 \\
& C_{N}^{i}=\frac{1}{|N|}\left[C(N)-\sum_{i=1}^{n} \sum_{\substack{S \subseteq N-\{i\} \\
S \ni \mathrm{i}}} C_{S}^{i}\right] \quad i=1, \ldots, 3 \\
& \text { et } C_{S}^{i} \geq 0
\end{aligned}
$$$$
\text { Alors } \operatorname{VS}(i)=\sum_{\substack{S \subseteq N \\ \mathcal{S} \rightarrow i}} C_{S}^{i}
$$$$
\text { et } \sum_{i=1}^{3} V S(i)=C(N)
$$

où $C(\cdot)$ est la fonction de coût et $V S(\cdot)$ la valeur de Shapley pour la commune $i^{10}$.

10. Signalons que cette démarche est généralisable au cas où $n>3$ mais que la valeur résultante ne correspondrait plus à la valeur de Shapley. 


\section{A.2.3 Un exemple d'échange d'énergie électrique}

Le problème analysé par Breton et Haurie [7] est de comment répartir les réductions de coûts engendrées par la coopération, prenant la forme d'échanges, entre 1'Hydro-Québec et PASNY ${ }^{11}$, deux firmes productrices d'énergie électrique et se trouvant chacune dans une situation de monopole sur son marché. Étant donné que seule l'Hydro-Québec (producteur 2) peut avoir des quantités excédentaires, les échanges peuvent se produire selon les deux scénarios suivants:

(i) Le producteur 1 (PASNY) demande au producteur 2 de lui fournir une quantité $y^{*}$. Le producteur 2 accepte à condition de recevoir la totalité de la réduction du coût de production de PASNY qui est égale à :

$$
C_{1}\left(x_{1}\right)-C_{1}\left(x_{1}-y^{*}\right)
$$

où $C_{1}\left(x_{1}\right)$ est le coût minimal pour produire $x_{1}$ et $C_{1}(\cdot)$ est une fonction de coût convexe et différentiable.

(ii) Dans le deuxième scénario, c'est le producteur 2 qui offre $y^{*}$ et le producteur 1 accepte de l'acheter à condition de ne débourser que le coût additionnel, encouru par 2, et occasionné par la production de $y^{*}$. Soit,

$$
C_{2}\left(x_{2}+y^{*}\right)-C_{2}\left(x_{2}\right)^{12}
$$

En supposant ces deux scénarios équiprobables, chaque producteur recevrait le paiement moyen :

$$
\pi=1 / 2\left(C_{1}\left(x_{1}\right)-C_{1}\left(x_{1}-y^{*}\right)+C_{2}\left(x_{2}+y^{*}\right)-C_{2}\left(x_{2}\right)\right)
$$

Pour étendre cette approche normative de partage des gains résultant de la coopération (ou échanges) au cas où le nombre de producteurs impliqués serait égal à 3 , les auteurs ont suivi le cheminement adopté par Shapley pour déterminer la valeur d'un jeu coopératif sous forme caractéristique. Soit les quantités :

$$
\begin{array}{ll}
R(\{1,2,3\}) & =\text { réduction du coût total de production quand les trois joueurs } \\
& \text { coopèrent } \\
R(\{i, j\}) & =\text { réduction du coût de production quand } i \text { et } j \text { coopèrent } \\
& (i \neq j, i, j \in\{1,2,3\}) \\
R(\{j\}) & =0
\end{array}
$$

$R$ (.) définit la fonction caractéristique du jeu d'échange. Le paiement moyen attribué au joueur $j$ est alors donné par la valeur de Shapley:

$$
W_{j}^{*}=\sum_{s \subset\{1,2,3\}} \frac{(s-1) !(n-s) !}{\mathrm{n} !}[R(S)-R(S-\{j\})]
$$

On vérifie aisément que

$$
\sum_{j=1}^{3} W_{j}^{*}=R(\{1,2,3\})
$$

11. Power Authority of the State of New York.

12. $C_{2}\left(x_{2}\right)$ et $C_{2}($.$) sont définies d'une façon analogue à C_{1}\left(x_{1}\right)$ et $C_{1}(\cdot)$. 


\section{B. La tarification d'un service}

\section{B.1 Le problème}

La tarification d'un service offert par un monopole public a retenu durant ces dernières années une attention particulière de la part des économistes et a donné naissance à une controverse qui puise ses sources dans les spécificités d'un tel service:

(i) Sorenson et al. [35] soutiennent que dans les industries d'utilité publique (électricité, téléphone, eau...) le coût marginal $\left(c_{m}\right)$ est souvent décroissant et, par conséquent, une tarification au $c_{m}$, prôné par le modèle de concurrence parfaite, engendre un déficit qui devrait être financé par des subventions gouvernementales. Ce fait implique que des non-usagers payent pour les usagers du service. Par conséquent, cette tarification est injuste (ou non équitable) à moins de supposer que son objectif est de procéder à une redistribution des revenus afin de maximiser une certaine fonction de bien-être collectif. Hazlewood [15], dans un article traitant de la tarification des services téléphoniques, refutait cet argument en soutenant que l'objectif premier d'une firme publique est d'offrir un service de la façon la plus efficace et la plus équitable. Si la notion d'efficacité se traduit dans ce contexte d'une part par une production à coût minimal et, d'autre part, par une structure tarifaire qui assure la couverture des coûts plus un certain rendement sur capital investi, la notion d'équité est plus difficile à délimiter (j'y reviendrai).

(ii) Les services publics possèdent généralement la caractéristique d'indivisibilité (Lœhman et Whinston [18]) qui signifie que l'utilisation d'un facteur de production dépend de la décision d'offrir ou de ne pas offrir le service en question et non de son niveau. Par exemple, si on veut relier plusieurs villes par un réseau ferroviaire, il faut placer des rails indépendamment du nombre de trains qui circuleront par la suite. Cette indivisibilité a des répercussions, via la décroissance dans les coûts, sur la sélection d'une politique de tarification.

(iii) Le manque d'homogénéité dans la demande peut engendrer des externalités pécuniaires. Pour illustrer ce point, supposons l'existence de deux usagers d'un certain service, le premier ayant une demande très élevée par rapport à celle exprimée par le deuxième. Sous l'hypothèse de décroissance du $c_{m}$, la demande du deuxième consommateur peut être satisfaite à un coût (unitaire) moins élevé que celui payé par le premier ${ }^{13}$. De ceci découle que les prix ne sont pas donnés (c'est une hypothèse du modèle de concurrence) mais sont plutôt fonction de l'ordre dans lequel les demandes s'expriment et de leurs niveaux.

13. Si on accepte l'hypothèse que chaque consommateur doit débourser au moins le coût occasionné par sa demande. 
(iv) La dernière caractéristique, qui va de soi, est l'impossibilité d'offrir un tel service sur une base individuelle.

Tenant compte de ces éléments, plusieurs auteurs se sont attaqués au problème d'établir une procédure de tarification équitable. Nombre d'entre eux l'ont formulé en termes d'un jeu coopératif et ont proposé la valeur de Shapley comme solution. Dans les deux sections suivantes, on analysera l'approche méthodologique adoptée pour passer par la suite aux applications empiriques.

\section{B.2 L'axiomatique de la tarification fondée sur la VS}

Le premier pas à franchir dans la recherche d'une tarification équitable est de précisément définir cette notion d'équité. Pour le faire, certains économistes ont énoncé un nombre de critères (sous forme d'axiomes) qui découlent de règles de «bon sens ». La deuxième étape étant la mise au point d'une formule mathématique reflétant les critères énumérés.

Littlechild [17] a suggéré quatre critères (axiomes) «naturels » pour définir l'équité :

(i) chaque groupe de consommateurs doit couvrir les coûts occasionnés par sa demande ;

(ii) les consommateurs obtenant le même service payeront le même tarif ;

(iii) la tarification doit être une fonction non décroissante de la demande;

(iv) si certains consommateurs procurent un bénéfice quelconque à d'autres, ils payeront moins.

Le premier critère garantit que des usagers ne subventionnent pas d'autres usagers et que les non-utilisateurs ne subventionnent pas les consommateurs du service. D'autre part, si on accepte l'hypothèse de décroissance du $c_{m}$ et vu le manque d'homogénéité dans la demande, il devient clair qu'une tarification au $c_{m}$ ne peut être équitable dans la mesure où deux consommateurs exprimant la même demande peuvent être amenés à payer des tarifs différents, dépendamment du moment où ils se décident à partager le service avec les autres consommateurs. Le but du deuxième axiome est précisément d'éliminer ce genre de situation. Pour satisfaire au moins les deux premiers critères de l'équité, les économistes ont proposé l'idée d'une tarification qui serait basée uniquement sur le coût additionnel (incremental cost) occasionné par la demande d'un certain groupe de consommateurs et qui soit indépendante de celle des autres. Lœhman et Whinston [18 et 19] ont repris cette idée et l'ont définie rigoureusement par une série de cinq axiomes.

\section{B.2.1 Les axiomes de Lœhman et Whinston}

Soit $N$ l'ensemble des utilisateurs et $G$ un sous-ensemble de $N$. Désignons par $C\left(K_{G}\right)$ le coût minimum pour satisfaire la demande $K_{G}$ de $G$. Le coût additionnel causé par la demande d'un consommateur $i \in G$ est alors défini par :

$$
\rho(G, i)=C\left(K_{G}\right)-C\left(K_{G-i}\right)
$$


Signalons que $C\left(K_{G}\right)$ est positive si $K_{G}$ l'est.

Axiome 1: La couverture des coûts totaux $C\left(K_{N}\right)$

$$
\sum_{i \in N} C(i)=C\left(K_{N}\right)
$$

où $C(i)$ est le tarif (ou le prix) que devrait payer le consommateur $i$.

Axiome 2: $C(i)$ doit dépendre uniquement du coût additionnel occasionné par la demande de $i$ et non des coûts induits par celle des autres. Formellement, soit $G_{1}, \ldots, G_{2} n-1$ un arrangement quelconque de tous les sous-groupes $G \subseteq N$ et contenant $i$, alors

$$
C(i)=F_{i}\left(\rho\left(G_{1}, i\right), \rho\left(G_{2}, i\right), \ldots, \rho\left(G_{2} n-1, i\right)\right)
$$

et $C(i)$ est indépendant de $\rho(G, j)$ pour $i \neq j, \forall G \subseteq N$.

Axiome 3: Le tarif est indépendant de l'ordre dans lequel les demandes s'expriment et ne dépend que de leurs niveaux. Formellement, $F_{i}($. $) \equiv F_{j}(.) \forall i, j \in N$.

Axiome 4: Le prix est une fonction homogène de degré un:

$$
\begin{aligned}
& F_{i}\left(\lambda \rho\left(G_{1}, i\right), \ldots, \lambda \rho\left(G_{2} n-1, i\right)\right)=\lambda F_{i}\left(\rho\left(G_{1}, i\right), \ldots, \rho\left(G_{2} n-1, i\right)\right) \\
& \forall \lambda \geq 0 .
\end{aligned}
$$

Cette propriété implique que $F_{i}(0, \ldots, 0)=0$.

Axiome 5: $F_{i}$ est deux fois continûment différentiable.

Sous les hypothèses (axiomes) 1 à 5 , et pour des demandes $K_{1}, \ldots, K_{n}$ fixées et positives, le prix est donné par:

$$
C(i)=\sum_{G \subseteq N} \frac{(n-g) !(g-1) !}{n !}\left[C\left(K_{G}\right)-C\left(K_{G-i}\right)\right]
$$

où $g$ et $n$ désignent respectivement le nombre d'éléments de $G$ et de $N$.

$C$ (i) ainsi défini n'est rien d'autre que la valeur de Shapley pour la fonction caractéristique $C($.$) . L'intérêt majeur des axiomes de Lœhman et Whinston$ réside dans la justification de la $V S$ en termes économiques propres au problème de tarification équitable. Dans le même ordre d'idée, Bös et Tilmann [6], Mirman et Tauman [23] et Samet et Tauman [26] ont proposé aussi une approche axiomatique pour caractériser ce qui est convenu d'appeler les prix Aumann-Shapley, une notion dérivée de la valeur d'un jeu non atomique définie par Aumann et Shapley et présentée plus haut ${ }^{14}$.

14. Billera et Heath [3] et Billera, Heath et Verrecchia [5] ont aussi élaboré, dans une optique comptable, un ensemble d'axiomes caractérisant les prix Aumann-Shapley. Ils seront analysés dans la section réservée à ce thème. 


\section{B.2.2 L'axiomatique des prix Aumann-Shapley ${ }^{15}$}

Le dénominateur commun des travaux cités ci-haut est de caractériser un mécanisme de prix (à définir) pour répartir les coûts de production, basé uniquement sur les fonctions de coûts et sur les quantités produites, sans faire aucune allusion aux utilités des consommateurs. Rappelons tout d'abord que la valeur Aumann-Shapley (VAS) d'un jeu non atomique est donnée par:

$$
\phi(f \circ \mu)(S)=\sum_{j=1}^{n} \mu j(S) \int_{0}^{1} \frac{\delta f}{\delta x_{j}}(t \mu(I)) d t
$$

Billera et al. [4] ont donné le nom de prix Aumann-Shapley $(P A S)$ à la quantité $\int_{0}^{1} \frac{\delta f}{\delta x_{j}}(t \mu(I)) d t$ qu'on va déterminer axiomatiquement.

Soit $F^{m}$ l'ensemble de toutes les fonctions $F$ à valeurs réelles définies sur $E_{+}^{m}$ (espace euclidien de dimension $m$ ) satisfaisant $F(0)=0$ et qui sont continûment différentiables sur $E_{+}^{m}$. Un producteur est caractérisé par une fonction de coût $F \in F^{m}$ définie sur $E_{+}^{m} . F(\alpha)$ est le coût encouru pour produire $\alpha$. Un mécanisme de prix est une fonction $P$ qui associe à chaque $F \in F^{m}$ et à chaque $\alpha$ dans $E_{+}^{m}$ un vecteur $P(F, \alpha)$ dans $E^{m}$ pour tout $m$ :

$$
P(F, \alpha)=\left(P_{1}(F, \alpha), \ldots, P_{m}(F, \alpha)\right) .
$$

Axiome 1: Changement d'échelle.

Soit $F \in F^{m}$ et $\lambda_{1}, \ldots, \lambda_{m} m$ nombres réels positifs. Soit $G \in F^{m}$ définie par:

$$
G\left(x_{1}, \ldots, x_{m}\right)=F\left(\lambda_{1} x_{1}, \ldots, \lambda_{m} x_{m}\right)
$$

Alors, pour tout $\alpha \in E_{+}^{m}$ et $\forall i(i=1, \ldots, m)$

$$
P_{i}(G, \alpha)=\lambda_{i} P_{i}\left(F\left(\lambda_{1} \alpha_{1}, \ldots, \lambda_{m} \alpha_{m}\right)\right) \text {. }
$$

En d'autres termes, si on change l'échelle de mesure d'un bien (de kilogramme en tonne par exemple) on aura un changement équivalent dans son prix.

Axiome 2: Cohérence.

Soit $F \in F^{m}$ et $G \in F^{1}$. Si pour $\forall x \in E_{+}^{m}$

$$
F\left(x_{1}, \ldots, x_{m}\right)=G\left(\sum_{i=1}^{m} x_{i}\right)
$$

Alors, pour chaque $i(i=1, \ldots, m)$ et pour chaque $\alpha \in E_{+}^{m}$,

$$
P_{i}(F, \alpha)=P\left(G, \quad \sum_{i=1}^{m} \alpha_{i}\right)
$$

15. Les notations sont celles de Samet et Tauman [26]. 
Cet axiome stipule que si le coût de production d'un produit $A$ est le même que celui de produire un bien $B$, le prix sera le même pour $A$ et $B$, indépendamment de l'utilité du consommateur pour $A$ et $B$.

Axiome 3: Additivité

Soit $F, G$ et $H$ des fonctions dans $F^{m}$. Si pour $\forall x \in E_{+}^{m}$ on a :

$$
F\left(x_{1}, \ldots, x_{m}\right)=G\left(x_{1}, \ldots, x_{m}\right)+H\left(x_{1}, \ldots, x_{m}\right)
$$

Alors, pour tout $\alpha \in E_{+}^{m}$,

$$
P(F, \alpha)=P(G, \alpha)+P(H, \alpha)
$$

Axiome 4: Positivité

Soit $F \in F^{m}$ et $\alpha \in E_{++}^{m}(\alpha>0)$. Si $F$ est une fonction non décroissante pour tout $x \leq \alpha$, alors

$$
P(F, \alpha) \geq 0
$$

La forme particulière des mécanismes de prix qui satisfont les axiomes 1 à 4 est établie par le théorème suivant:

Théorème: $P(.,$.$) satisfait les axiomes 1$ à 4 si et seulement si, il existe une mesure non négative $\mu$ sur $([0,1], \beta)(\beta$ est la tribu des Boréliens de $[0,1])$ telle que pour tout $m, \forall F \in F^{m}$ et $\forall \alpha \in E_{+}^{m} \alpha \neq 0$,

$$
P_{i}(F, \alpha)=\int_{0}^{1} \frac{\delta f}{\delta x_{i}}(t \alpha) d \mu(t)
$$

Pour interpréter intuitivement $P_{i}(F, \alpha)$, supposons que $\alpha$ est produit d'une façon homogène débutant à zéro et se terminant à $\alpha$. Supposons aussi qu'à chaque instant une fraction infinitésimale est produite. Le prix de cette fraction n'est alors rien d'autre que son coût marginal et le prix de $\alpha$ est égal à la moyenne pondérée par $\mu$ de ses coûts marginaux. Deux cas particuliers présentent un grand intérêt:

(i) $\quad \operatorname{si} \mu(\{1\})=1$ alors, $P_{i}(F, \alpha)=\frac{\delta F}{\delta x_{i}}$

ce qui, en d'autres termes, signifie que le prix de la commodité est égal à son coût marginal ;

(ii) si $\mu$ est la mesure de Lebesgue sur $[0,1]$ alors,

$$
P_{i}(F, \alpha)=\int_{0}^{1} \frac{\delta F}{\delta x_{i}}(t \alpha) d t,
$$

ce qui correspond au mécanisme de prix Aumann-Shapley. 
Ce deuxième résultat peut être obtenu, comme l'ont fait Mirman et Tauman [23] et Billera et Heath [3], d'une autre façon qui consiste à ajouter à la liste des axiomes deux autres qui s'énoncent comme suit:

Axiome 5: La couverture des coûts totaux

$$
\alpha \cdot P(F, \alpha)=F(\alpha)
$$

En d'autres termes, le revenu total doit être égal au coût total, ce qui peut se traduire par une absence de subventions.

Axiome 6: Bien factice.

Cet axiome stipule que si un bien $i(i=1, \ldots, m)$ n'a pas d'incidence sur les coûts alors son prix est nul.

Mirman et Tauman ont alors démontré que le seul mécanisme de prix satisfaisant les 6 axiomes est celui de Aumann-Shapley. D'autre part, moyennant une prise en compte explicite des utilités des consommateurs, ils ont prouvé l'existence d'un équilibre pour ce mécanisme de prix. Par ailleurs, Bös et Tillmann [6] ont étendu cette approche en considérant le cas où la firme est sujette à une réglementation gouvernementale qui se traduit par une contrainte du type:

$$
\alpha \cdot P(F, \alpha)=k \cdot F(\alpha) \quad k>0(*)
$$

Pour $k=1, P(.,$.$) est le mécanisme de prix Aumann-Shapley. Généralement$ (pour $k>0$ ), $P(.,$.$) prend la forme suivante:$

$$
P(F, \alpha)=\int_{0}^{1} \frac{\delta F}{\delta x}(t \alpha) k d \lambda(t)
$$

où $\lambda$ est la mesure de Lebesgue. Notons que pour prouver que ce mécanisme de prix est compatible avec la demande formulée par 1 consommateurs, il faut incorporer $(*)$ dans le modèle, en plus de tenir compte des utilités des consommateurs.

\section{B.3 Quelques exemples d'applications empiriques}

Pour illustrer l'utilisation du mécanisme de prix Aumann-Shapley, on a sélectionné deux articles traitant deux problèmes différents; la tarification des services téléphoniques à l'université Cornell et la répartition des coûts de transport entre plusieurs destinations.

\section{B.3.1 Calcul des tarifs téléphoniques à l'université Cornell}

L'article de Billera, Heath et Raanan [4] constitue une des premières tentatives de résolution d'un problème réel de tarification avec les ingrédients de la théorie des jeux non atomiques et plus précisément la notion de valeur d'un jeu (valeur Aumann-Shapley). Le problème considéré se résume en la recherche de prix 
équitables pour partager les coûts téléphoniques, fournis globalement, de l'université Cornell entre les différents usagers. Pour cela, les auteurs ont défini un jeu non atomique appelé le «jeu téléphonique » dont la description suit.

$\mathrm{Vu}$ que le tarif d'un appel interurbain dépend de la destination (il y en a $k$ ), de la tranche horaire ( 24 par jour) ainsi que de la journée (jours ouvrables versus fin de semaine) on aura en tout $n=24 \times k \times 2$ types d'appels. Soit $(I, C)$ un espace mesurable où $I=[0, n]$ et $C$ la tribu des Boréliens de $[0, n]$. Le sous-intervalle $I_{j}=[j-1, j]$ représente les appels du type $j$. On définit sur $I$ des mesures $\mu_{j}(j=1, \ldots, n)$ tels que $\mu_{j}=\alpha_{j} \lambda\left(S \cap I_{j}\right)$ où $\lambda$ est la mesure de Lebesgue et $\alpha_{j}$ est le nombre total de minutes d'appel du type $j$ pour la période (mois) en question. $\mu_{j}(S)$ représente le nombre total de minutes d'appel du type $j$ pour le sous-ensemble $S$. Soit $\mu=\left(\mu_{1}, \ldots, \mu_{n}\right)$ un vecteur de mesures et soit $f\left(x_{1}, \ldots, x_{n}\right)$ le coût minimal (incluant les frais généraux) pour satisfaire la charge $X=\left(x_{1}, \ldots, x_{n}\right)$.

À partir de là, les auteurs sont en mesure de définir un jeu $v$ sur $I$ par $v=f o \mu$ tel que pour tout $S \in C, v(S)=f(\mu(S))$ ce qui correspond au coût minimal pour satisfaire la demande de $S$. Comme on l'a déjà signalé, la valeur d'AumannShapley d'un jeu non atomique $v$ est donnée par:

$$
\phi(f \circ \mu)(S)=\sum_{j=1}^{n} \mu_{j}(S) \int_{0}^{1} f_{j}(t \mu(I)) d t
$$

où $f_{j}=\frac{\delta f}{\delta x_{j}}$ et $\phi(f \circ \mu)(S)$ représente la part du coût total qui revient à la coalition $S$.

D'autre part, si $S=I_{k}$ alors $\mu_{j}\left(I_{k}\right)=0$ sauf si $j=k$ et, par conséquent,

$$
\phi(f \circ \mu)\left(I_{k}\right)=\mu_{k}\left(I_{k}\right) \quad \int_{0}^{1} f_{k}(t \mu(I)) d t
$$

ce qui correspond au coût total de tous les appels de type $k$. Vu que $\mu_{\mathrm{k}}\left(I_{k}\right)$

représente le nombre total de minutes d'appel de type $k$, alors $\mu_{k}=\int_{0}^{1} f_{k}(t \mu(I)) d t$

n'est rien d'autre que le tarif, par minute, de l'appel de type $k$. Notons pour terminer que Billera et al. fournissent les résultats numériques obtenus. 


\section{B.3.2 Un problème d'allocation des coûts de transport}

S'inspirant de cet article de Billera et al., Samet, Tauman et Zang [27] ont proposé les prix (ou les tarifs) Aumann-Shapley pour allouer les coûts unitaires d'un vecteur de commodités circulant sur un réseau de transport. Le problème découle du fait que généralement on minimise le coût global pour l'ensemble du réseau, ce qui peut induire que certaines destinations soient amenées à payer un tarif plus élevé que son coût minimum. Par conséquent, et comme l'illustre l'exemple suivant, une certaine injustice se crée.

Soit le réseau de transport décrit par les éléments suivants :

(i) il existe deux origines $B_{1}$ et $B_{2}$ où des ressources $b_{1}$ et $b_{2}$ sont disponibles;

(ii) il existe deux destinations $A_{1}$ et $A_{2}$ où des quantités $x_{1}$ et $x_{2}$ sont demandées ;

(iii) soit $c_{i j}$ le coût de transport unitaire de l'origine $B_{i}(i=1,2)$ à la destination $A_{j}(j=1,2)$;

(iv) la solution optimale de ce problème de minimisation du coût global est donnée par le programme suivant:

$$
\begin{aligned}
& F\left(x_{1}, x_{2}\right)=\min \sum_{n, m} c_{n m} y_{n m} \\
& \text { sujet à } \quad \sum_{n=1}^{2} y_{n m}=x_{m} \quad m=1,2 \\
& \sum_{m=1}^{2} y_{n m} \leq b_{n} \quad n=1,2 \\
& y_{n m} \geq 0
\end{aligned}
$$

(v) considérons le cas particulier où,

$$
\begin{aligned}
& b=\left(b_{1}, b_{2}\right)=(20,20) \\
& x=\left(x_{1}, x_{2}\right)=(20,20) \\
& C=\left(\begin{array}{cc}
10 & 15 \\
1000 & 1500
\end{array}\right)
\end{aligned}
$$

On vérifie facilement que la solution optimale consiste à transporter 20 unités de $B_{2}$ à $A_{1}$ avec un coût de $20 \times 1000=20000$ et à envoyer 20 unités de $B_{1}$ à $A_{2}$ avec un coût de $20,15=300$. Le coût total serait égal à 20300 . Ce faisant, on empêche la destination $A_{2}$ de se procurer les 20 unités demandées au coût le plus faible (10) ce qui est en quelque sorte injuste. Pour remédier à ce problème, les auteurs proposent d'allouer le coût minimal total entre les différentes destinations $m(m=1, \ldots, M)$ selon le mécanisme de prix Aumann-Shapley qui s'écrit :

$$
P(F, \alpha)=\left(P_{1}(F, \alpha), \ldots, P_{M}(F, \alpha)\right)
$$

où $P_{m}(F, \alpha)=\int_{0}^{1} \frac{\delta F}{\delta x_{m}}(t \alpha) d t$ 
Par ailleurs, les auteurs démontrent que ce mécanisme est le seul à satisfaire les cinq axiomes ${ }^{16}$ dont il a été question à la section précédente et ceci pour certains types de fonctions de coût.

\section{La comptabilisation des coûts conjoints}

L'allocation des coûts conjoints occupe une place importante dans la littérature comptable. Sous l'impulsion de l'article de Shubik ${ }^{17}$ auquel on a déjà eu l'occasion de se référer et de celui de Mossin [24] où l'auteur propose le noyau et la valeur de Shapley ( $V S$ ) comme mécanismes de répartition des profits résultant de la fusion entre deux ou plusieurs firmes, une partie de cette littérature s'est alors édifiée autour des différents types de solutions d'un jeu coopératif et plus particulièrement le noyau et la VS. Étant donné qu'au niveau de la formulation formelle, il n'existe aucune différence entre le traitement du problème de la comptabilisation des coûts conjoints et celui réservé au partage des coûts d'investissements publics ou au partage des coûts d'un service (étudiés respectivement dans les sections $\mathrm{A}$ et $\mathrm{B}$ ), on se contentera d'une très courte section pour rendre compte de ces recherches où l'accent sera mis sur le vocabulaire comptable.

Deux types de situations où des coûts conjoints sont présents ont préoccupé la littérature comptable où la théorie des jeux coopératifs intervient:

(i) dans toute organisation décentralisée, il existe un certain nombre de facilités communes à tous les départements (ou même aux centres de profits) tels que les ressources informatiques ou les services d'achats, etc. Le problème est alors de trouver un mécanisme d'allocation $(M A)$ des coûts de ces services entre les différents départements. Hamlen et al. [12] soutiennent que tout $M . A$ est en quelque sorte arbitraire et, par conséquent, en choisir un revient à évaluer, sur la base d'un certain nombre de critères jugés «importants », les $M$. $A$ disponibles. Si le coût marginal de production (d'un service quelconque) est décroissant, la firme a intérêt à inciter ses départements à se procurer le service chez le même producteur. Pour le faire, il faudrait que chaque département ou coalition de départements débourse moins de ce qu'il devrait payer s'il décidait d'acquérir le service par ses propres moyens. On reconnaît ici les conditions du noyau d'un jeu coopératif où les départements forment l'ensemble des joueurs et où la fonction caractéristique $V(S)$ du jeu est déterminée par les économies de coûts disponibles aux départements appartenant à la coalition $S$. Une fois que l'ensemble des imputations appartenant au noyau est délimité, on peut envisager plusieurs possibilités pour en choisir une. Si l'équité figure sur la liste des bonnes propriétés d'un $M$.A et si la VS est dans le noyau, alors son choix paraît naturel. Hamlen et al. ont

16. Répartition des coûts, positivité, additivité, cohérence et changement d'échelle. [33].

17. «Incentives, Decentralized Control, the Assignment of Joint Costs and Internal Pricing», 
généralisé dans [13] la VS comme mécanisme d'allocation des coûts conjoints en relaxant tour à tour les axiomes qui la déterminent. Callen [8] l'a proposé (la $V S$ ) pour comptabiliser l'apport relatif d'un investissement au profit engendré par plusieurs investissements interdépendants et synergiques;

(ii) la plupart des processus de production sont multi-produits et nécessitent généralement plusieurs inputs. Si la fonction de coût $f\left(x_{1}, \ldots, x_{n}\right)$ est séparable i.e. $f\left(x_{1}, \ldots, x_{n}\right)=\sum_{i=1}^{n} f_{i}\left(x_{i}\right)$, alors l'allocation d'un coût unitaire à chaque produit $i$ ne présente aucune difficulté (coût unitaire du produit $\left.i=f_{i}\left(x_{i}\right) / x_{i}\right)$. Si tel n'est pas le cas, la question qui se pose alors est de savoir quelle partie de $f\left(x_{1}, \ldots, x_{n}\right)$ affectée à la production d'une unité du produit $i$ (un exemple fort connu de ce type de fonctions est la fonction de production Cobb-Douglas $\left.f(K, L)=A K^{\alpha} L^{1-\alpha}\right)$. Billera et Heath [3] et Billera, Heath et Verrecchia [5] ont répondu à cette question en proposant le mécanisme (ou procédure) d'allocation Aumann-Shapley (longuement analysé à la section B) qu'ils ont déterminé par quatre axiomes (répartition des coûts, additivité, monotonie et cohérence) et en supposant d'une part que la fonction $f$ est continûment dérivable et, d'autre part, l'absence de coûts fixes i.e. $f(0, \ldots, 0)=0$. Rappelons que la formule explicite de ce mécanisme d'allocation s'écrit :

$$
C_{i}=\int_{0}^{1} \frac{\delta f}{\delta x_{i}}\left(t \alpha_{1}, \ldots, t \alpha_{n}\right) d t
$$

où $C_{i}$ est le coût unitaire de $i$.

\section{CONCLUSION}

L'objectif premier de ce travail était de mettre en évidence l'utilité de la théorie des jeux coopératifs comme cadre conceptuel pour approcher les problèmes reliés à la répartition équitable de ressources communes à plusieurs agents économiques. L'accent a été mis sur une technique particulière, la valeur de Shapley, proposée à l'origine comme solution a priori d'un jeu coopératif. On a montré, à travers une revue de la littérature, la diversité des problématiques qui ont été abordées en ayant recours à cette notion de valeur d'un jeu qui, rappelons-le, détermine un partage unique, Pareto-optimal et surtout équitable. Pour terminer, notons que le seul élément qui pourrait limiter l'applicabilité de la $V S$ à un problème quelconque de partage provient de l'hypothèse qui stipule que les coalitions se forment d'une façon aléatoire, hypothèse qui, il va de soi, n'est pas toujours respectée dans la réalité. 


\section{BIBLIOGRAPIHE}

[ 1] Aumann, R.J., Shapley, L.S., Values of Non-Atomic Games, Princeton University Press, N.J., 1974.

[ 2] Berge, C., Théorie générale des jeux à n personnes, Mémorial des sciences mathématiques, Fascicule CXXXVIII, 1957.

[ 3] Billera, L.J., Heath, D.C., «Allocation of Shared Costs : a Set of Axioms Yielding a Unique Procedure », Mathematics of Operations Research, vol. 7, $\mathrm{n}^{\circ} 1$, février 1982.

[ 4] Billera, L.J., Heath, D.C., Raanan, J., «Internal Telephone Billing Rates - A Novel Application of Non-Atomic Game Theory », Operations Research, vol. 26, ${ }^{\circ} 6$, novembre - décembre 1978.

[ 5] Billera, L.J., Heath, D.C., Verrecchia, R.E., «A Unique Procedure for Allocating Common Costs from a Production Process », Journal of Accounting Research, vol. 19, ${ }^{\circ} 1$, printemps 1981.

[ 6] Bös, D., Tillmann, G., «Cost-Axiomatic Regulatory Pricing», Journal of Public Economics, 22, pp. 243-256, 1983.

[ 7] Breton, A., Haurie, A., «Modèles de gestion à équipement fixé dans le secteur de l'électricité en vue de l'application aux échanges d'énergie », Actes du colloque sur la théorie des systèmes et la gestion scientifique des services publics, 1977.

[ 8] Callen, J.L., «Financial Cost Allocations : a Game Theoretic Approach », The Accounting Review, vol. LIII, $\mathrm{n}^{\circ} 2$, avril 978.

[ 9] Ekeland, Y., La théorie des jeux et ses applications à l'économie mathématique, P.U.F., 1974.

[10] FaUlhaber, G.R., ZaJAC, E.E., «Some Thoughts on Cross-Subsidization: Notes for a Talk for the International Conference on Telecommunications Economics », Bell Laboratories Economic Discussion Paper, $\mathrm{n}^{\circ} 48$, janvier 1976.

[11] Gately, D., «Sharing the Gains from Regional Cooperation: a Game Theoretic Application to Planning Investment in Electric Power », International Economic Review, 15 (1), pp. 195-208, février 1974.

[12] Hamlen, S.S., Hamlen, W.A., Tschirhart, J.T., «The Use of Core Theory in Evaluating Joint Cost Allocation Schemes », The Accounting Review, vol. LII, $\mathrm{n}^{\circ} 3$, juillet 1977.

[13] Hamlen, S.S., Hamlen, W.A., Tschirhart, J.T., «The Use of the Generalized Shapley Allocation in Joint Cost Allocation », The Accounting Review, vol. $\mathrm{LX}, \mathrm{n}^{\circ} 2$, avril 1980 .

[14] HaURIE, A., «Game Theory and Management of Complex Systems », Forthcoming in Encyclopedia of Systems and Control. 
[15] Hazlewood, A., "Optimum Pricing as Applied to Telephone Service», The Review of Economic Studies, 18 (46), pp. 67-78, 1951.

[16] Jones, A.J., Game Theory, Hellis Horwood, Chichester, W. Sussex, 1980.

[17] Littlechild, S.C., «A Game-Theoretic Approach to Public Utility Pricing », Western Economic Journal, 8 (2), pp. 162-166, juin 1970.

[18] Loehman, E., Whinston, A., "A New Theory of Pricing and Decision Making for Public Investment », Bell Journal of Economics, 2 (2), pp. 606-625, automne 1971.

[19] Loehman, E., Whinston, A., «An Axiomatic Approach to Cost Allocations for Public Investments », Public Finance Quartely, vol. 2, n² 2, avril 1974.

[20] Luce, R.D., RaIfFa, H., Games and Decisions, Wiley, 1957.

[21] McKinseY, J.C.C., Introduction to the Theory of Games, McGraw-Hill, New York, 1952.

[22] Megiddo, N., «Computational Complexity of the Game Theory Approach to Cost Allocation for a Tree », Mathematics of Operations Research, vol. 3, $\mathrm{n}^{\circ} 3$, août 1978 .

[23] Mirman, L.J., Tauman, Y., «Valeur de Shapley et répartition équitable des coûts de production », Cahiers du Séminaire d'Économétrie, 23, pp. 121151 .

[24] Mossin, J., «Merger Agreements: Some Game-Theoretic Considerations », The Journal of Business, 41, pp. 460-471, octobre 1968.

[25] Rawls, J., A Theory of Justice, Cambridge, Mass., 1971.

[26] Samet, D., Tauman, Y., «The Determination of Marginal Cost Prices under a Set of Axioms», Econometrica, vol. 50, $\mathrm{n}^{\circ}$ 4, juillet 1982.

[27] Samet, D., Tauman, Y., Zang, I., «An Application of the Aumann-Shapley Prices for Cost Allocation in Transportation Problems », Mathematics of Operations Research, vol. 9, n 1, février 1984.

[28] Schleicher, H., «Techniques de répartition des coûts dans l'évaluation des investissements publics : une analyse par la théorie des jeux », Economies et Sociétés, vol. 14, mai - décembre 1980.

[29] Schmeidler, D., "The Nucleolus of a Characteristic Function Form Game », SIAM J. Appl. Math., 17 (6), pp. 1163-1170, novembre 1969.

[30] Schotter, A., Schwödiauer, G., «Economics and the Theory of Games: a Survey », Journal of Economic Literature, vol. 18, pp. 479-527, juin 1980.

[31] Shapley, L.S., «A Value for n-Person Games», Annals of Mathematics Studies, $\mathrm{n}^{\circ} 28$, Contributions to the Theory of Games, vol. II, Edited by H.W. Kuhn and A.W. Tucker, Princeton University Press, Princeton, N.J., pp. 307-317, 1953.

[32] Shapley, L.S., « Cores of Convex Games », International Journal of Game Theory, vol. I, pp. 11-26, 1971. 
[33] Shubiк, M., «Incentives, Decentralized Control, the Assignment of Joint Costs and Internal Pricing », Management Science, 8 (3), pp. 325-343, avril 1962.

[34] Shubiк, M., Game Theory in the Social Sciences, The MIT Press, Cambridge, Mass., 1982.

[35] Sorenson, J., Tschirhart, J., Whinston, A., «A Theory of Pricing under Decreasing Costs ", The American Economic Review, vol. 68, ${ }^{\circ} 4$, septembre 1978.

[36] Spinetto, R., «Fairness in Cost Allocations and Cooperative Games », Decision Sciences, vol. 6, pp. 482-491, 1975.

[37] Von Neumann, J., Morgenstern, O., Theory of Games and Economic Behavior, Princeton University Press, Princeton, N.J., 1944.

[38] Vorob'ev, N.N., Game Theory, Lectures for Economists and Systems Scientists, Springer-Valag (1977), New York. 